\title{
Targeting CDK7 increases the stability of Snail to promote the dissemination of colorectal cancer
}

\author{
Yan Zhou ${ }^{1} \cdot$ Linlin $\mathrm{Lu}^{1} \cdot$ Guanmin Jiang ${ }^{2,3} \cdot$ Zhuojia Chen $^{4} \cdot$ Jiexin $\mathrm{Li}^{1} \cdot$ Panpan $\mathrm{An}^{1} \cdot$ Likun Chen $^{4} \cdot$ Jun Du${ }^{1}$. \\ Hongsheng Wang $\mathbb{D}^{1}$
}

Received: 29 May 2018 / Revised: 9 September 2018 / Accepted: 8 October 2018 / Published online: 19 November 2018

(c) ADMC Associazione Differenziamento e Morte Cellulare 2018

\begin{abstract}
Targeted inhibition of cyclin-dependent kinase 7 (CDK7) via its covalent inhibitor THZ1 can suppress the growth of various cancers, while its roles on colorectal cancer (CRC) remain obscure. Here we report that the expression of CDK7 is upregulated in CRC cells and tissues. THZ1 exhibits high potency and selectivity against CRC cells both in vitro and in vivo via induction of cell apoptosis rather than cell cycle disruption. Intriguingly, THZ1 treatment increases the ability of epithelial mesenchymal transition (EMT) and in vivo metastasis to liver of CRC cells. Mechanistical studies reveal that THZ1 increases the expression of Snail, while not other EMT-transcription factors, via enhancing its protein stability rather than mRNA expression or translation. By screening Snail stability related factors via qRT-PCR, results indicate THZ1 and si-CDK7 decrease the expression of protein kinase D1 (PKD1) in CRC cells. Down regulation of PKD1 mediates THZ1 up regulated Snail via dephosphorylation of Snail Ser 11 and prevention of proteasome mediated degradation. Clinical analysis confirms that $C D K 7$ is significantly $(p<0.05)$ negatively correlated with the expression of mesenchymal markers including FN1, VIM, and MMP2. CRC patients whose tumors expressing less CDK7/SNAI1 or PKD1/SNAI1 showed significant $(p<0.05)$ poorer overall survival (OS) rate as compared with those with greater levels. Collectively, our data suggest that targeted inhibition of CDK7 can trigger the metastasis of CRC during cancer development via PKD1/Snail axis, which imposes great challenge that inhibition of CDK7 is a potential approach for cancer treatment.
\end{abstract}

These authors contributed equally: Yan Zhou, Linlin Lu, Guanmin Jiang.

Edited by G. Del Sal

Electronic supplementary material The online version of this article (https://doi.org/10.1038/s41418-018-0222-4) contains supplementary material, which is available to authorized users.

\section{Hongsheng Wang}

whongsh@mail.sysu.edu.cn

hongshengwang@foxmail.com

1 Department of Microbial and Biochemical Pharmacy, School of Pharmaceutical Sciences, Sun Yat-sen University, Guangzhou 510006, China

2 Department of Clinical Laboratory, The Fifth Affiliated Hospital, Sun Yat-sen University, Zhuhai, Guangdong 519000, China

3 Central Laboratory, The Fifth Affiliated Hospital, Sun Yat-sen University, Zhuhai, Guangdong 519000, China

4 Sun Yat-sen University Cancer Center; State Key Laboratory of Oncology in South China; Collaborative Innovation Center for Cancer Medicine, Guangzhou 510060, China

\section{Introduction}

Colorectal cancer (CRC) is the second most common cause of cancer death in the world, which responsible for $\sim 655,000$ deaths globally each year [1]. As the major metastasis organ, synchronous liver metastases exist in up to $20 \%$ of newly diagnosed CRC patients [2], and only $13 \%$ of 5-year survival rate of CRC patients has been reported [3]. Luckily, due to numerous studies revealing alterations in oncogenes and tumor suppressor genes contributed to the tumorigenesis and development of CRC, the clinical treatment and drug targeted therapies of CRC have been greatly improved over the last decades [4]. However, the detailed mechanisms underlying CRC progression and metastasis are still far from being completely elucidated.

Cyclin-dependent kinases (CDKs), the critical regulatory enzymes controlling all cell cycle transitions, have been suggested as the potent therapeutic targets for cancers [5]. Among the identified CDKs, CDK7 has a general role in the phosphorylation of the RNA polymerase II carboxyterminal domain and therefore regulates the initiation and elongation of 


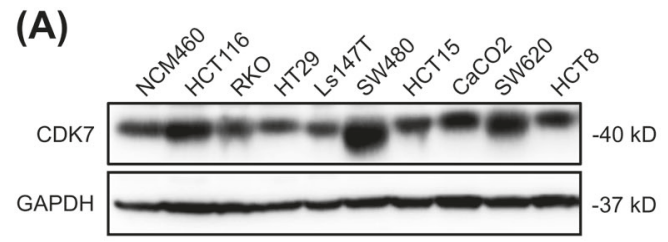

(B)

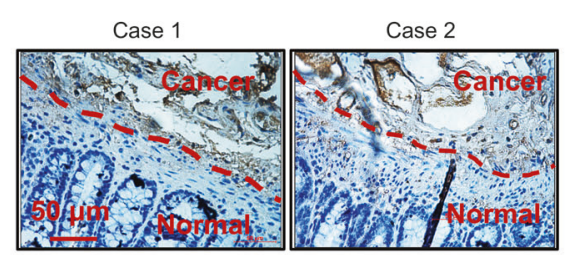

(C)

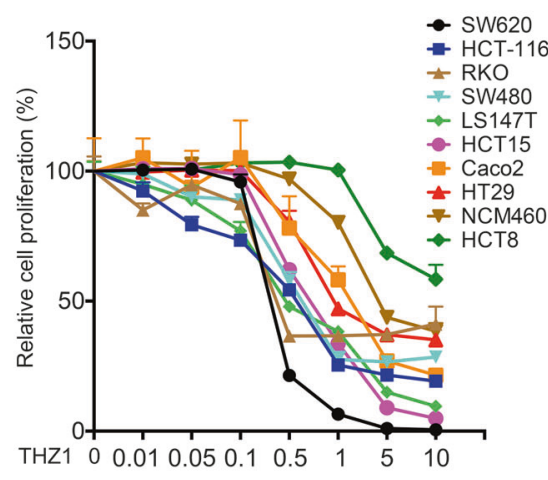

\begin{tabular}{|c|c|}
\hline Cell line & IC $50(\mu \mathrm{M})$ \\
\hline SW620 & 0.312 \\
\hline HCT-116 & 0.491 \\
\hline LS147T & 0.556 \\
\hline HCT15 & 0.792 \\
\hline SW480 & 0.988 \\
\hline RKO & 1.207 \\
\hline Caco2 & 1.982 \\
\hline HT29 & 2.327 \\
\hline NCM460 & 4.964 \\
\hline HCT8 & $>10$ \\
\hline
\end{tabular}

(E)

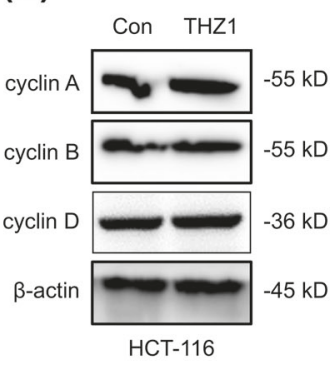

(G)

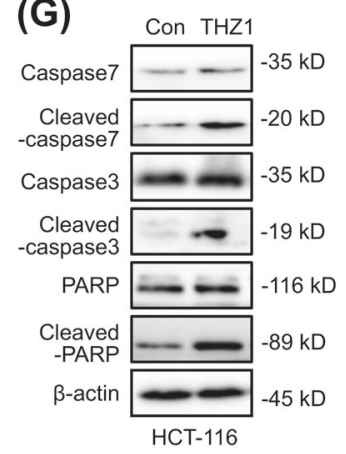

(H)

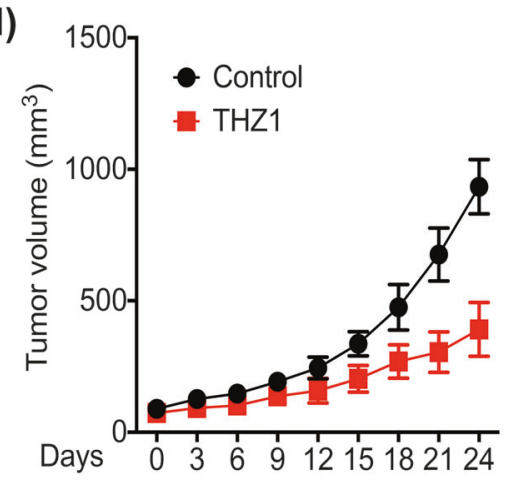

(I)

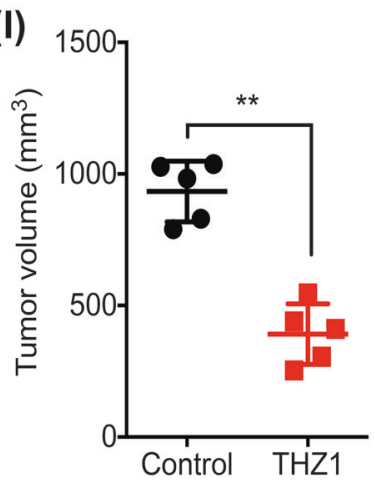

(J)

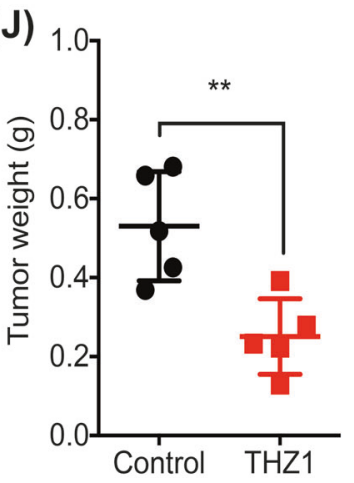

transcription [6, 7]. Although the detailed mechanisms remain poorly understood, inhibition of the CDK7 can regulate Pol II promoter occupancy $[8,9]$, promoter-proximal pausing $[10$ 12] and mRNA stability [13]. Targeted inhibition of CDK7 via a newly developed covalent inhibitor THZ1 can negatively impact gene expression, suppress the proliferation of cancer cells, and inhibit progression of animal xenograft models [10, 12, 14, 15].

There are only limited data about the expression of CDK7 and its roles in the progression of CRC. Selective 
Fig. 1 THZ1 exhibited high potency to suppress growth of CRC cells. a The expression of CDK7 in CRC and human colonic epithelial NCM460 cells was checked by western blot analysis; b Typical IHC staining for CDK7 expression in tumor tissues from CRC patients (c) Dose-response curves (left) and $\mathrm{IC}_{50}$ values (right) of $\mathrm{CRC}$ and human colonic epithelial NCM460 cells after treated with increasing concentrations of THZ1 for $48 \mathrm{~h}$; d, e HCT-116 cells were synchronized at the G1/S transition by a double TdR block, and then treated with 100 nM THZ1 for indicated time periods. The cell cycles were analyzed by FCM (d), and cell cycle related proteins were measured by western blot analysis (e); f, g HCT-116 cells were treated with increasing concentrations of THZ1 for $48 \mathrm{~h}$, stained with annexin V-FITC and PI, and then analyzed by FCM for cell apoptosis (f). Apoptotic proteins were measured by western blot analysis $(\mathbf{g})$. $\mathbf{h}$ Tumor growth curves of HCT-116 cells in xenograft models treated with or without THZ1 at indicated time intervals; $i, j$ Tumor volumes (i) and weights (j) of HCT-116 cells in xenograft models treated with or without THZ1 at the end of experiment; Data were presented as means \pm SD from three independent experiments

inhibition of CDK7 can block the activation of Cdk1 and Cdk2 and cause arrest at both $\mathrm{G}_{1} / \mathrm{S}$ and $\mathrm{G}_{2} / \mathrm{M}$ transitions of colon cancer HCT-116 cells [16]. Resveratrol can induce $\mathrm{G}_{2}$ arrest in colon carcinoma HT29 cells through inhibition of CDK7 [17]. Our present study revealed that targeted inhibition of CDK7 can suppress the proliferation of CRC cells; however, it increased the in vitro and in vivo metastasis of CRC cells via upregulation of Snail, a key transcription factor of epithelial-mesenchymal transition (EMT).

\section{Results}

\section{THZ1 exhibited high potency to suppress growth of CRC cells}

CDK7 can be widely detected in all measured 9 CRC cell lines and human colonic epithelial NCM460 cells (Fig. 1a). The expression of CDK7 in HCT-116, SW480 and SW620 CRC cells were much higher than that in human colonic epithelial NCM460 cells (Fig. 1a). IHC analyses by use of tissue microarrays demonstrated the expression of CDK7 in CRC tumor was significantly $(p<0.05)$ greater than that in the adjacent normal mucosal tissues (Fig. 1b, Table S1). Data set obtained from the Gene Expression Profiling Interactive Analysis (GEPIA) online database (http://gepia. cancer-pku.cn) showed that CDK7 expression was higher in CRC compared with that in other tumor types (Figure S1A). In addition, TCGA data confirmed that the expression of CDK7 in colon and rectal carcinoma (COAD, READ), diffuse large B-cell lymphoma, pancreatic cancer (PAAD) and thyroid carcinoma (THYM) tissues were significantly $(p<0.05)$ greater than that in the adjacent normal tissues (Figure S1B).
Effects of CDK7 on growth of CRC cells were analyzed by treating cells with THZ1, a covalent CDK7 inhibitor [10]. The sensitivity of CRC cells to THZ1 were much higher than that of human colonic epithelial NCM460 cells (Fig. 1c). The $\mathrm{IC}_{50}$ value of THZ1 for NCM460 cell was greater than other nine tested CRC cell lines, except HCT8 (Fig. 1c). The suppression effects of THZ1 on proliferation of CRC and NCM460 cells were confirmed by the cell morphology (Figure S1C) and colony formation assay (Figure S1D). We measured the effects of THZ1 on cell cycle progression. Surprisingly, THZ1 treatment did not alter the cell cycle of HCT-116 (Fig. 1d) or SW480 (Figure S1E) cells. Further, THZ1 had no effect on the expression of cyclin A/B/D in HCT-116 (Fig. 1e) or SW480 (Figure S1F) cells.

We then investigated the effect of THZ1 on cell apoptosis. THZ1 can increase the apoptosis of HCT-116 cells via a concentration-dependent manner (Fig. 1f). Consistently, THZ1 treatment induced the PARP cleavage and activation of caspases 7 and caspases 3 (Fig. 1g). Similar effects of THZ1 on apoptosis (Figure S1G) and cleavage of PARP and caspase-3 (Figure S1H) in SW480 cells were observed.

Next, we examined the effects of THZ1 on growth of HCT-116 tumor xenografts in nude mice. At the end of experiment, tumor sizes, volumes and weights in THZ1injected group were significantly lower than control group (Fig. 1h, i and j). Decreased Ki67-positive and increased cleaved caspase-3-positive cells in THZ1 group were detected in vivo (Figure S1I). Collectively, these data suggested that THZ1 can selectively inhibit the in vitro and in vivo growth of CRC cells via induction of cell apoptosis.

\section{THZ1 induced EMT and in vivo metastasis of CRC cells}

We then evaluated the potential roles of THZ1 on in vitro motility of CRC cells. Firstly, both HCT-116 and SW480 cells were treated with or without $100 \mathrm{nM}$ THZ1 for 3 days, then cells withdrawn of THZ1 was resuspended and cultured for further assay. Results showed that both HCT-116 cells (Fig. 2a) and SW480 cells (Figure S2A) treated with THZ1 had significant greater migration abilities than control cells. Consistently, transwell assay confirmed that THZ1 treatment can increase the in vitro invasion of both HCT116 and SW480 cells (Fig. 2b). EMT has been considered as one of the most important step for cancer cell metastasis [18]. Western blot analysis showed that THZ1 treatment increased the expression of vimentin and fibronectin (FN), while decreased the expression of E-Cadherin (E-Cad) in both HCT-116 and SW480 cells via a concentrationdependent manner (Fig. 2c), suggesting that THZ1 may induce EMT of CRC cells. Similarly, knockdown of CDK7 (Figure S2 B) by siRNA can increase the expression of 
(A)

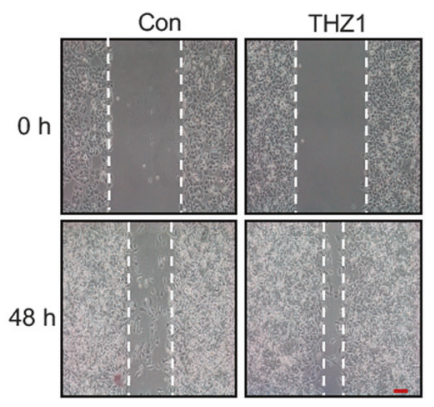

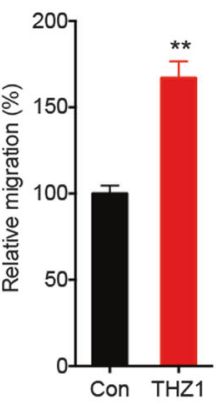

(B)

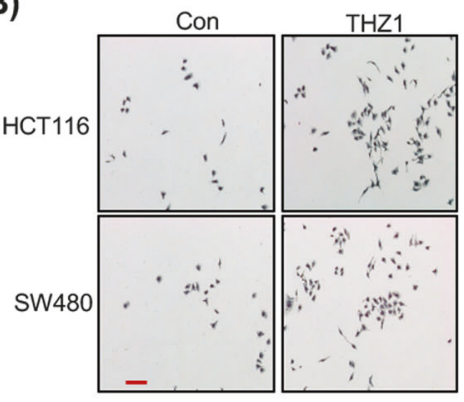

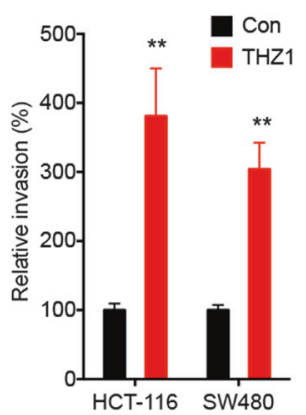

(C)

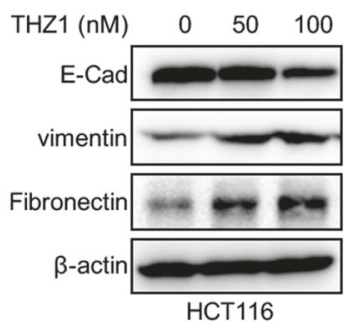

HCT116

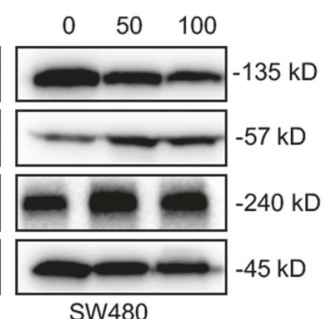

(D)

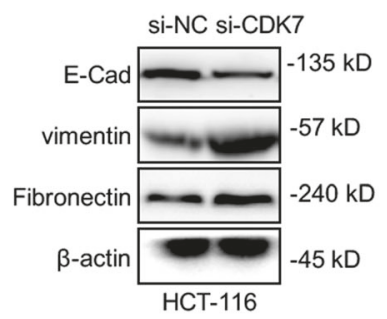

(E)
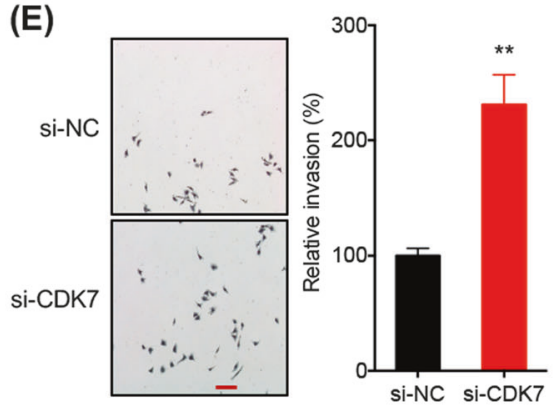

(F)

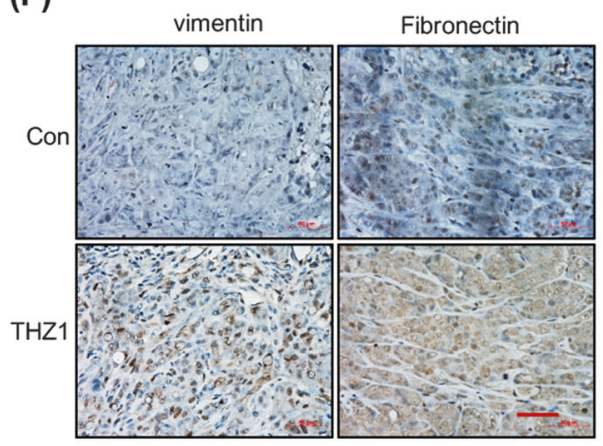

(G)

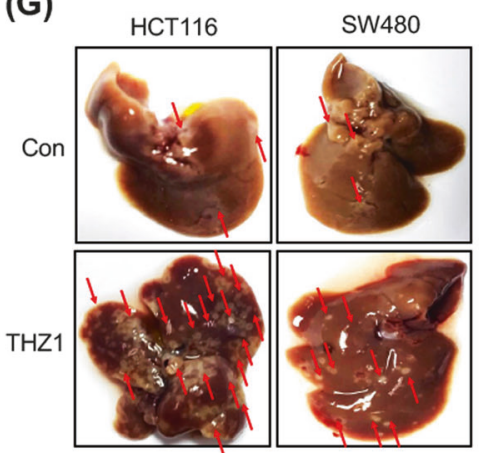

(H)

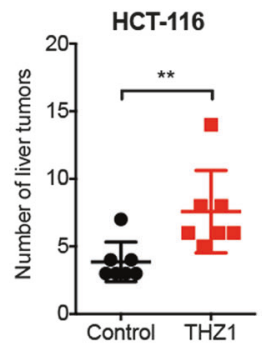

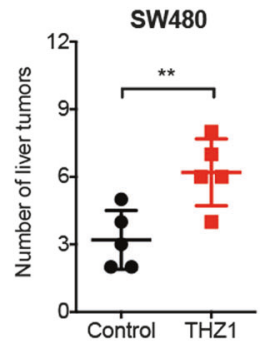

Fig. 2 THZ1 induced EMT and in vivo metastasis of CRC cells. a HCT-116 cells were treated with or without $100 \mathrm{nM}$ THZ1 for $48 \mathrm{~h}$, wound healing was recorded (left) and quantitatively analyzed (right); b HCT-116 or SW480 cells were treated with or without $100 \mathrm{nM}$ THZ1 for $48 \mathrm{~h}$. The in vitro invasion was tested by the use of CytoSelect ${ }^{\mathrm{TM}}$ 24-well Cell Invasion assay kits (left) and quantitatively analyzed (right); c HCT116 or SW480 cells were treated with or without THZ1 for $72 \mathrm{~h}$, protein levels of E-Cad, vimentin and Fibronectin were measured by western blot analysis; d, e After transfected with si-NC or si-CDK7 for $72 \mathrm{~h}$, protein levels of E-Cad, vimentin and fibronectin in HCT116 cells were measured by western blot analysis (d). The in vitro invasion ( $48 \mathrm{~h}$ ) was tested by use of CytoSelect ${ }^{\mathrm{TM}} 24-$ well Cell Invasion assay kits (left) and quantitatively analyzed (right) (e); f IHC (vimentin and fibronectin)-stained paraffin-embedded sections obtained from xenografts. Red bar $=50 \mu \mathrm{m} ; \mathbf{g}, \mathbf{h}$ HCT-116 or SW480 cells treated with or without THZ1 were injected into the nude mice by tail vein injection. Representative images of metastatic liver tumors were shown (left, g). Numbers of liver tumors derived from HCT-116 or SW480 cells treated with or without THZ1were quantitatively analyzed (h). Data were presented as means \pm SD from three independent experiments. Scale bar equals to $50 \mu \mathrm{m}, * p<0.05$; $* *<$ 0.01 compared with control vimentin while decrease the expression of E-Cad in HCT116 cells (Fig. 2d). Further, si-CDK7 increased the in vitro invasion of HCT-116 cells (Fig. 2e). Collectively, our data suggested that targeted inhibition of CDK7 induced the migration, invasion and EMT of CRC cells.

We examined the expression of EMT-related markers in THZ1-treated HCT-116 tumor xenografts in nude mice. IHC data showed that THZ1 increased the expression of vimentin and FN in HCT-116 tumor xenografts
(Fig. 2f). We further determined the impacts of THZ1 on in vivo metastasis of HCT-116 and SW480 cells. As shown in Fig. $2 \mathrm{~g}$, h, the numbers and sizes of liver tumors derived from THZ1-treated HCT-116 and SW480 cells were significantly increased compared to cells without treatment. Collectively, our data showed that targeted inhibition of CDK7 can enhance the in vitro and in vivo metastasis ability of CRC cells via induction of EMT. 
(A)

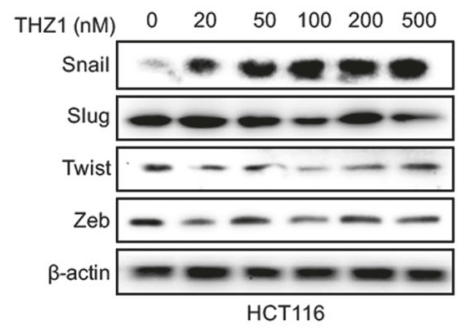

(C)

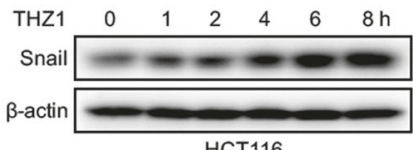

HCT116
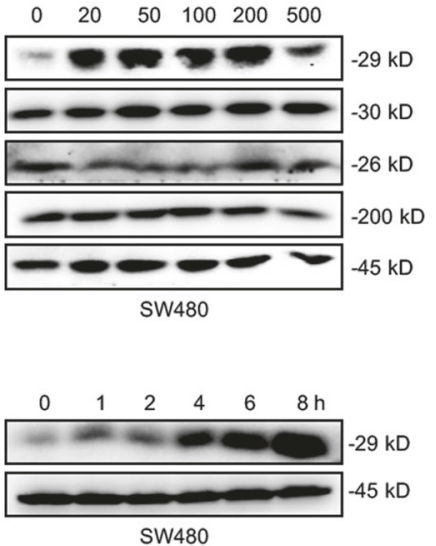

(B)

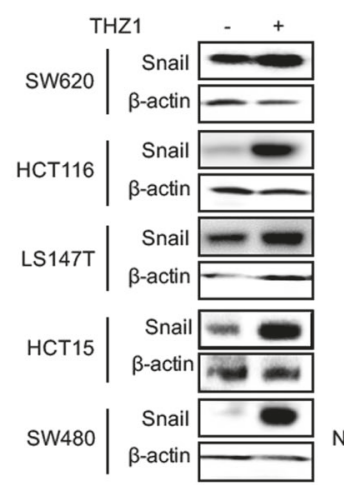

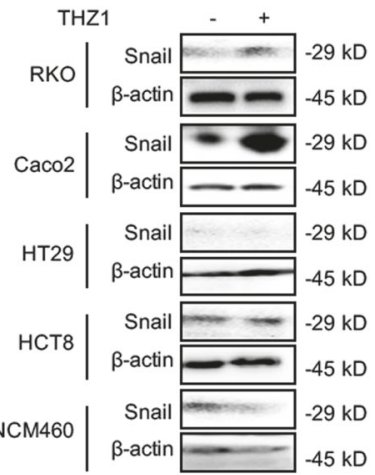

(D)

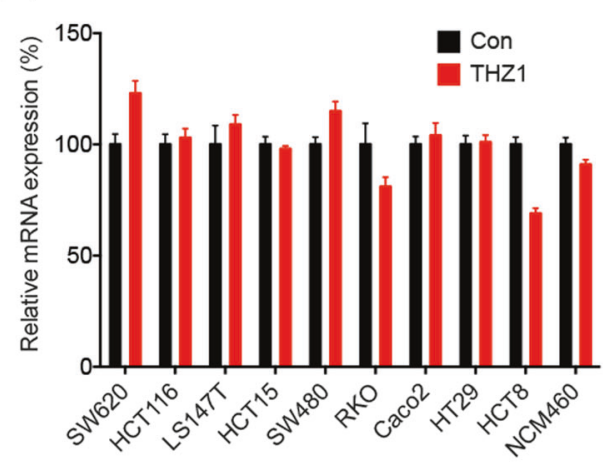

(H)

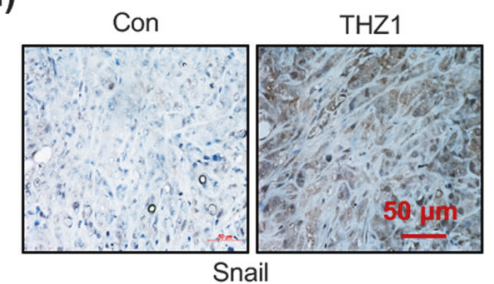

(E)

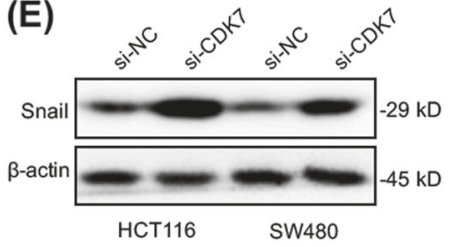

(G)

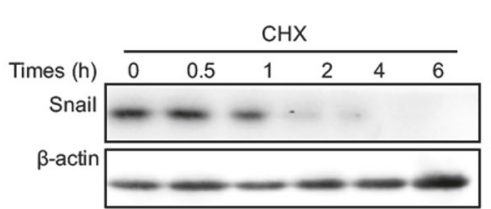

(F)

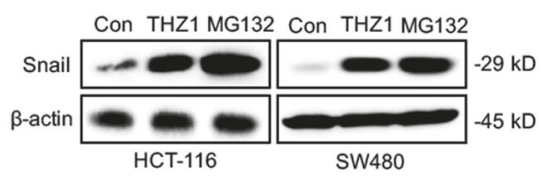

(I)

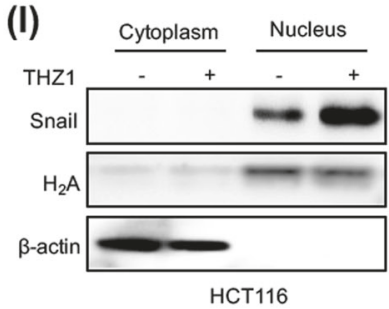

(J)
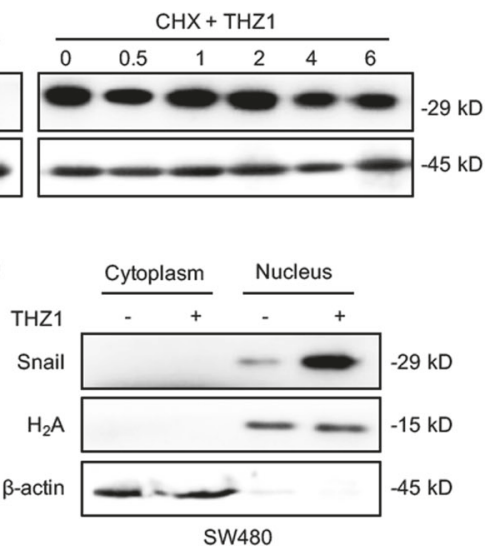

Fig. 3 Targeting CDK7 post-translationally increased Snail in CRC cells a HCT-116 or SW480 cells were treated with increasing concentrations of THZ1 for $4 \mathrm{~h}$, the expression of EMT-TFs was detected by western blot analysis; b CRC cells were treated with or without $100 \mathrm{nM}$ THZ1 for $4 \mathrm{~h}$, the expression of Snail was detected by western blot analysis; c HCT-116 or SW480 cells were treated with $100 \mathrm{nM}$ THZ1 for the indicated times, the expression of Snail was detected by western blot analysis; d CRC cells were treated with or without 100 $\mathrm{nM}$ THZ1 for $4 \mathrm{~h}$, the mRNA expression of Snail was detected by qRT-PCR; e HCT-116 or SW480 cells were transfected with si-NC or
si-CDK7 for $24 \mathrm{~h}$, the expression of Snail was detected by western blot analysis; f HCT-116 and SW480 cells were treated with $100 \mathrm{nM}$ THZ1 or MG132 $(10 \mu \mathrm{M})$ for $4 \mathrm{~h} ; \mathbf{g}$ HCT-116 cells were treated with $10 \mu \mathrm{g} /$ $\mathrm{ml}$ CHX combined with or without $100 \mathrm{nM}$ THZ1 for the indicated times; h IHC (Snail)-stained paraffin-embedded sections obtained from xenografts based on HCT-116 cells. Red bar $=50 \mu \mathrm{m} ; \mathbf{i}, \mathbf{j}$ After treated with $100 \mathrm{nM}$ THZ1 for $4 \mathrm{~h}$, the cytoplasm and nucleus of HCT-116 (i) or SW480 (j) cells were separated, and the expression of Snail was checked by western blot analysis. Data were presented as means \pm SD from three independent experiments

\section{Targeting CDK7 post-translationally increased Snail in CRC cells}

EMT-related transcription factors (EMT-TFs) such as Snail, Slug, Twist and Zeb1 can regulate progression of EMT via targeting E-cad expression [19]. Results showed that THZ1 dose-dependently increased the protein levels of Snail, but not Slug, Twist or Zeb1, in both HCT116 and SW480 cells after treatment for $6 \mathrm{~h}$ (Fig. 3a). Further, $100 \mathrm{nM}$ THZ1 was sufficient to increase the protein expression of Snail in most measured CRC cells, including HCT-116, SW480, Caco2, SW620, RKO and LS147T, while it had no significant effect in HT29, HCT8 or NCM460 cells (Fig. 3b). THZ1 treatment rapidly increased the protein expression of Snail 
in both HCT116 and SW480 cells after $2 \mathrm{~h}$ of incubation (Fig. 3c). However, the mRNA expression of all checked EMT-TFs including Snail, Slug, Twist and Zeb1 was no affected by THZ1 in either HCT116 or SW480 cells (Figure S3A). Similarly, THZ1 did not affect the mRNA expression of Snail in all detected CRC cells and human colonic epithelial NCM460 cells (Fig. 3d). Knockdown of CDK7 also increased the protein expression of Snail in both HCT116 and SW480 cells (Fig. 3e). However, transfection of si-CDK7 did not affect the mRNA expression levels of Snail in either HCT-116 or SW480 cells (Figure S3B).

The results indicated the rapid increase of protein levels of Snail might be due to post-translational regulation. Both HCT-116 and SW480 cells were incubated with proteasome inhibitor MG-132. Results showed that protein levels of MG132-treated Snail were comparable to those induced by THZ1 (Fig. 3f). Ubiquitylation of Snail is critical for its stabilization. Immunoprecipitation results showed that the relative ubiquitylation of Snail was dramatically decreased in THZ1-treated cells (Figure S3C). Further, THZ1 can extend the half-lives of Snail in both HCT116 (Fig. 3g) and SW480 (Figure S3C) cells. We further evaluated the effects of THZ1 on translation efficiency of Snail in CRC cells. We separated the RNA fractions to monosome translation initiation fraction (including non-translating fraction $<40 \mathrm{~S}$, 40S/60S ribosomes, and $80 \mathrm{~S}$ monosomes) as well as translation active polysome ( $>80 \mathrm{~S}$ ) (Figure S3E). qRT-PCR showed that THZ1 did not alter the monosome and polysome distribution of Snail mRNA in HCT-116 cells treated with or without THZ1 (Figure S3F).

In xenograft models based on HCT-116 cells, THZ1 treatment can also markedly increase the Snail expression in tumor tissues (Fig. 3h). The subcellular localization of Snail was further verified. Western blot analysis revealed that THZ1 treatment remarkably increased the nuclear localization of Snail in HCT-116 (Fig. 3i) and SW480 (Fig. 3j) cells. Collectively, targeted inhibition of CDK7 can increase the protein expression of Snail in CRC cells and tissues via stabilizing Snail protein, rather than increasing its mRNA expression or translation efficiency.

\section{Down regulation of PKD1 mediated THZ1 induced stabilization of Snail}

GSK-3 $\beta$ is a major kinase that phosphorylates Snail and induces its degradation [20], while activation of $\mathrm{NF}-\kappa \mathrm{B}$ induced COP9 signalosome 2 (CSN2) can block the ubiquitination and degradation of Snail [21]. Our data showed that the phosphorylation levels of GSK-3 $\beta$, p65 and Akt were not affected by THZ1 in either HCT-116 (Fig. 4a) or SW480 (Figure S4A) cells. Immunoprecipitation assay confirmed that CDK7, GSK-3 $\beta$ and CSN2 can directly bind to Snail in HCT-116 cells; however, these interactions were independent to THZ1 treatment in HCT-116 cells (Fig. 4b). Since GSK-3 $\beta$, p65, MAPK/ERK, PI3K/Akt, p38 and EGFR were important signals for the upregulation of Snail [22], we further pretreated cells with their corresponding inhibitors. No obvious effect of all tested inhibitors was observed in THZ1-treated HCT116 cells (Fig. 4c). It suggested that targeted inhibition of CDK7-induced stabilization of Snail regardless of the phosphorylated status of GSK-3 $\beta$ and NF- $\kappa$ B.

We then checked the mRNA expression of 27 factors that related to post-translational regulation of Snail stability or nuclear turnover of Snail (Summarized at Table S2). THZ1 treatment can significantly $(p<0.05)$ decrease the expression of PKD1, but had no significant effect on other factors, in both HCT-116 (Fig. 4d) and SW480 (Figure S4B) cells. Similarly, THZ1 can decrease the mRNA expression of PKD1 in both LS147T and RKO cells (Figure S4 C). Western blot analysis confirmed that THZ1 can decrease the expression of PKD1 in both HCT116 and SW480 cells (Fig. 4e). Inversely, over expression of CDK7 can increase the expression of PKD1 in both HCT116 and SW480 cells (Figure S4D). In xenograft models based on HCT116 cells, THZ1 treatment obviously decreased the PKD1 expression in tumor tissues (Fig. 4f).

To verify the roles of PKD1 in THZ1-induced upregulation of Snail, cells were transfected with PKD1 constructs (Fig. 4g). Results indicated that over expression of PKD1 not only decreased the expression of Snail but also completely abolished THZ1 induced upregulation of Snail (Fig. 4g). In addition, half-lives of Snail decreased when overexpressing PKD1 in HCT-116 (Fig. 4h) and SW480 (Figure S4E) cells. CID755673, the inhibitor of PKD1, can increase the expression of Snail in both HCT-116 and SW480 cells (Figure S4F), while CID755673 increased the protein stability of Snail in HCT-116 cells (Figure S4 G).

It was indicated that Ser 11 of Snail is phosphorylated by PKD1 in cancer cells [23]. We performed immunoprecipitation assay with anti-FLAG antibody to pull down Snail protein and examined its Ser-11 phosphorylation status by using an antibody that specifically recognizes p-Ser-11SNAIL. Our data showed that Snail protein presented a very strong protein band at the size of phosphorylated Snail, and it was strengthened in cells transfected with pcDNA/PKD1. However, treating cells with THZ1 or PKD1 inhibitor CID755673 resulted in a loss of phosphorylated Snail (Fig. 4i). To verify the roles of PKD1 and Ser 11 phosphorylation in CDK7-regulated Snail expression, we substituted Serine to Alanine at its Ser11 (pcDNA/Snail S11A) and Ser97/Ser101 (pcDNA/Snail 2SA). Our data showed that THZ1 increased the expression of pcDNA/Snail WT and pcDNA/Snail 2SA, while this effect was partially attenuated for pcDNA/Snail S11A (Fig. 4j). Further, THZ1 extended the half-life of wild type Snail, which was less 
(A)

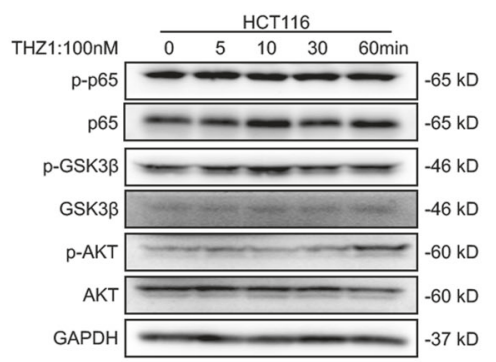

(C)

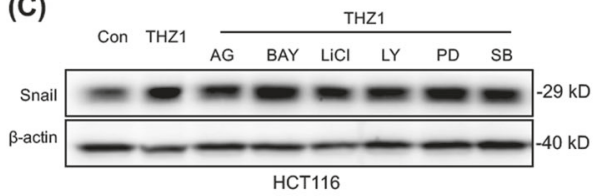

(G)

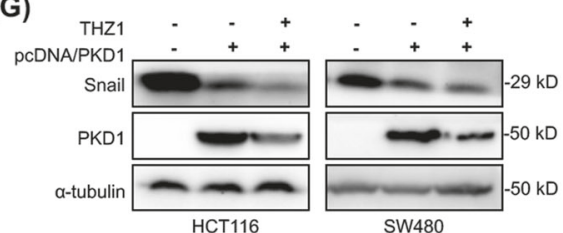

(B)

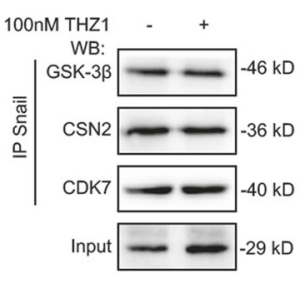

(D)

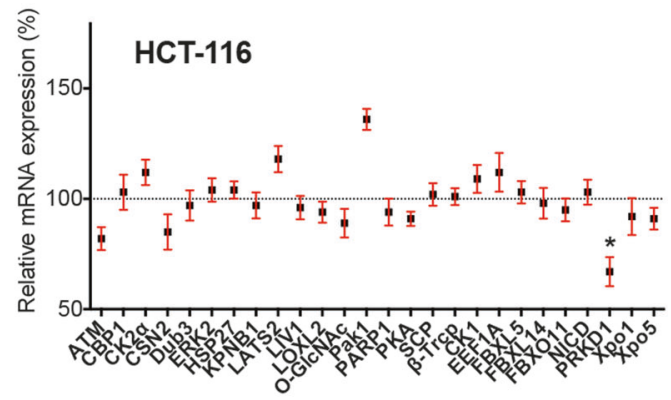

(E)

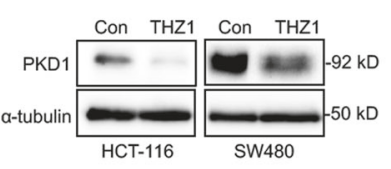

(I)

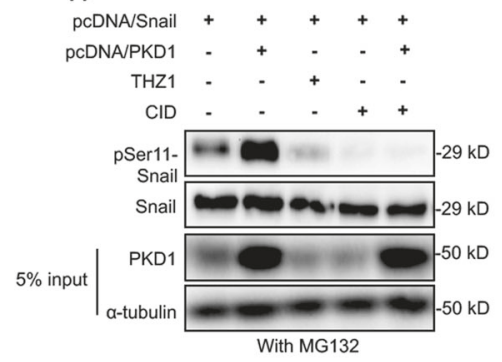

(F)

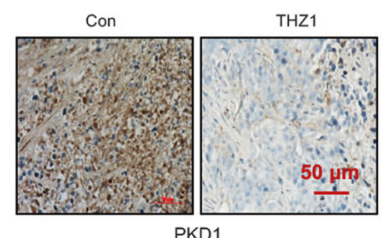

(J)

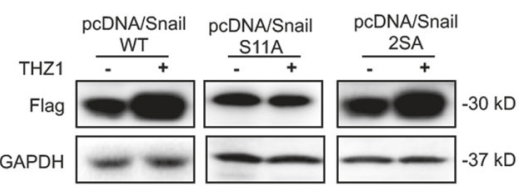

(H)
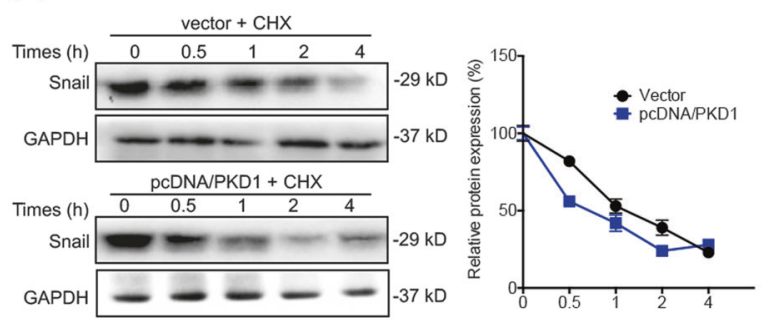

Fig. 4 Downregulation of PRKD1 mediated THZ1-induced stabilization of Snail. a HCT-116 cells were treated with $100 \mathrm{nM} \mathrm{THZ1} \mathrm{for} \mathrm{the}$ indicated times. Phosphorylated and total expression of GSK-3 $\beta$, p65 and Akt were detected; b HCT116 cells treated with or without 100 $\mathrm{nM}$ THZ1 for $4 \mathrm{~h}$, and Snail was immunoprecipitated for detection of GSK-3 $\beta$, CSN2, and CDK7; c HCT-116 cells were pretreated with

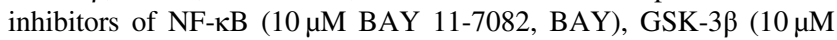
LiCl), PI3K/Akt (10 $\mu \mathrm{M}, \mathrm{LY} 294002, \mathrm{LY})$, ERK1/2 (10 $\mu \mathrm{M}$ PD98059, PD), EGFR (10 $\mu \mathrm{M}$ AG1478, AG) and p38 MAPK (10 $\mu \mathrm{M}$ SB203580, $\mathrm{SB}$ ) for $90 \mathrm{~min}$, respectively, followed by $4 \mathrm{~h}$ incubation of $100 \mathrm{nM}$ THZ1; d HCT-116 cells treated with or without $100 \mathrm{~nm}$ THZ1 for $4 \mathrm{~h}$, and mRNA expression levels of Snail stability-related factors were analyzed by qRT-PCR; e HCT-116 and SW480 cells were treated with or without $100 \mathrm{~nm}$ THZ1 for $4 \mathrm{~h}$. Protein levels of PKD1 were analyzed by western blot analysis; f IHC (Snail)-stained paraffinembedded sections obtained from xenografts based on HCT-116 cells. Red bar $=50 \mu \mathrm{m} ; \mathbf{g}$ After $24 \mathrm{~h}$ transfection of empty vector or

efficient for Snail S11A in HCT-116 cells (Fig. 4k). Similarly, CID755673 had no significant effect on the half-life of Snail S11A in HCT-116 cells, either (Figure S4H).
(K)
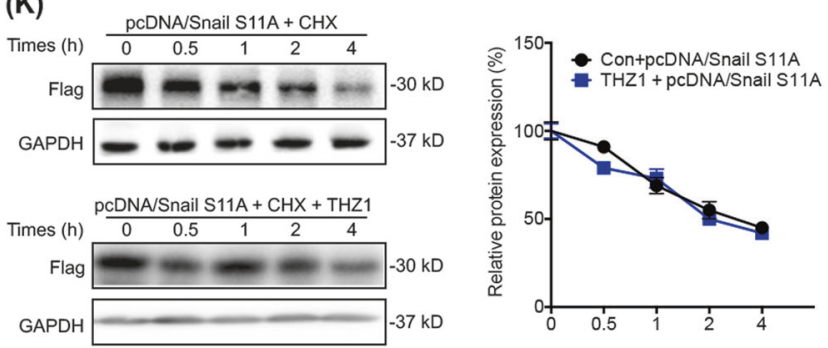

pcDNA/PKD1, HCT-116 or SW480 cells were treated with or without $100 \mathrm{~nm}$ THZ1 for $4 \mathrm{~h}$. Protein levels of Snail and PKD1 were analyzed by western blot analysis; $\mathbf{h}$ After $24 \mathrm{~h}$ transfection of empty vector or pcDNA/PKD1, HCT-116 cells were treated with $50 \mu \mathrm{g} / \mathrm{ml}$ CHX for the indicated times. Protein levels of Snail were analyzed by western blot analysis; i HCT-116 cells were treated as indicated, and further treated with MG132 for $6 \mathrm{~h}$ before co-IP experiments. Ser11 phosphorylation of immunoprecipitated Snail protein were detected; j After transfection of pcDNA/Snail WT, pcDNA/Snail S11A, or pcDNA/ Snail 2SA for $24 \mathrm{~h}, \mathrm{HCT}-116$ cells were further treated with or without $100 \mathrm{nM}$ THZ1 for $4 \mathrm{~h}$. Expression levels of Snail were analyzed by western blot analysis; $\mathbf{k}$ After transfection of pcDNA/Snail S11A for $24 \mathrm{~h}, \mathrm{HCT}-116$ cells were further treated with $50 \mu \mathrm{g} / \mathrm{ml} \mathrm{CHX}$ for the indicated times. Expression levels of Snail were analyzed by western blot analysis. Data were presented as means \pm SD from three independent experiments. $* * p<0.01$ compared with control

Collectively, our data suggested that downregulation of PKD1 mediated THZ1-induced Snail stabilization via dephosphorylation of Ser 11. 
(A)
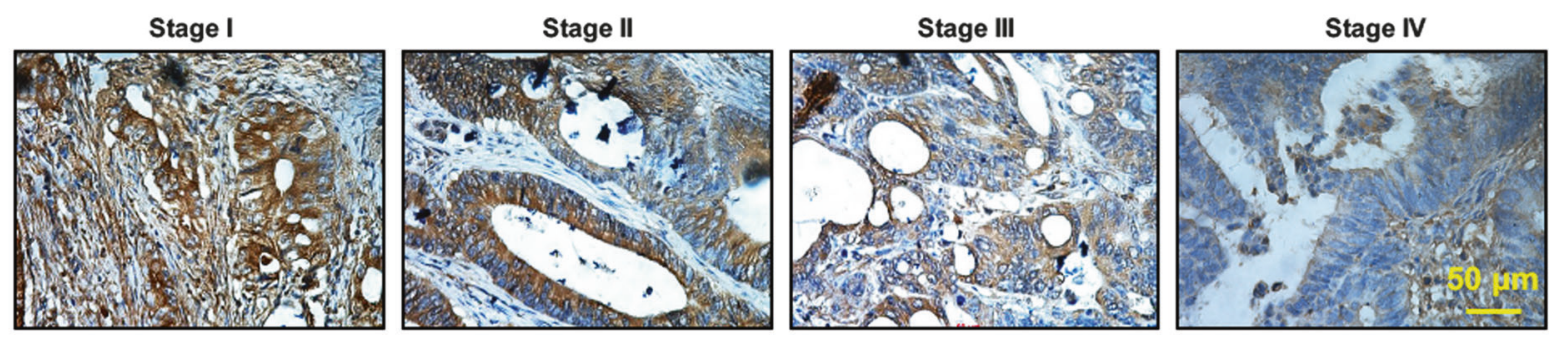

(B)

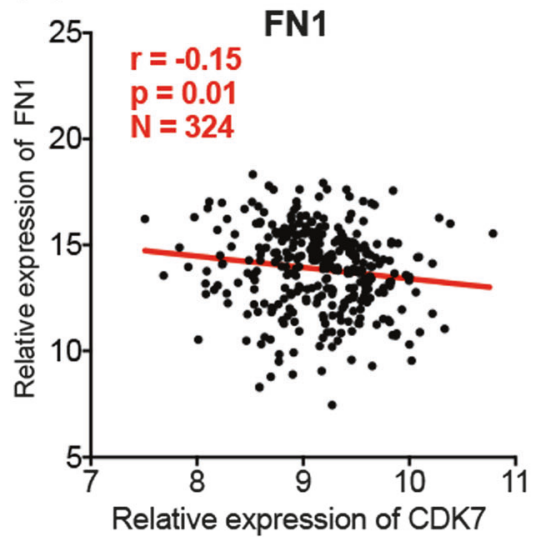

(E)

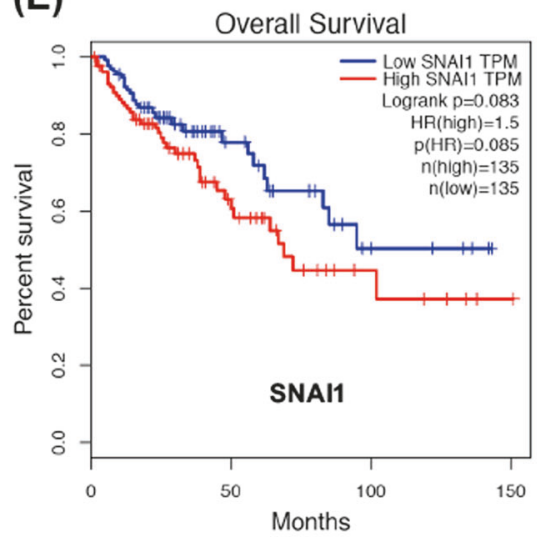

(C)

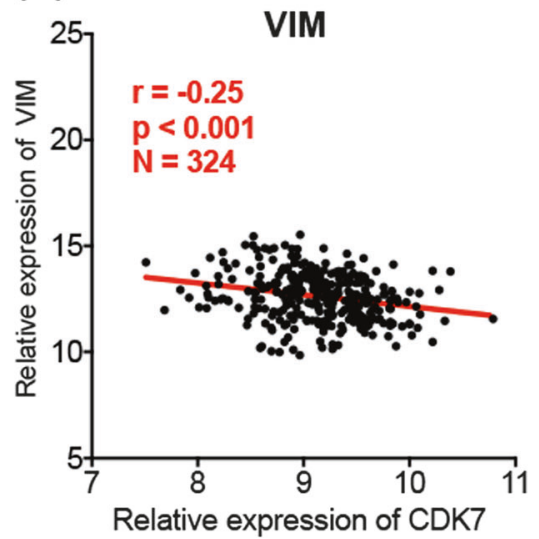

(F)

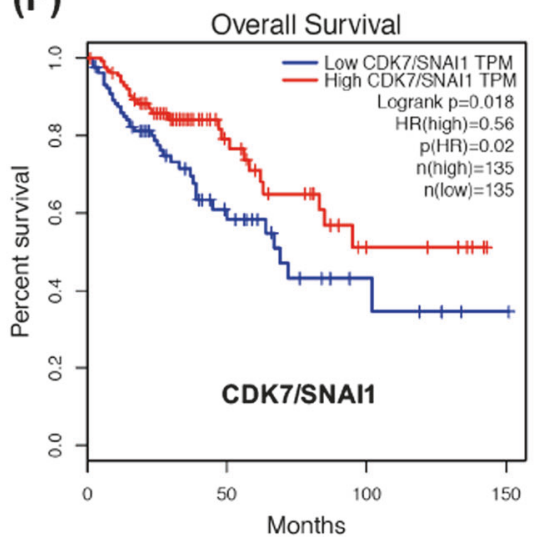

(D)

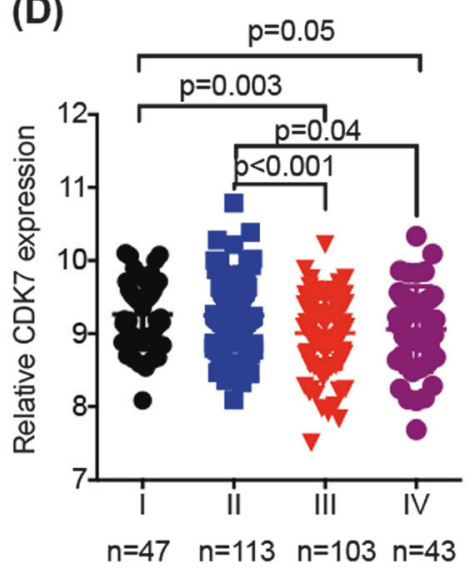

(G)

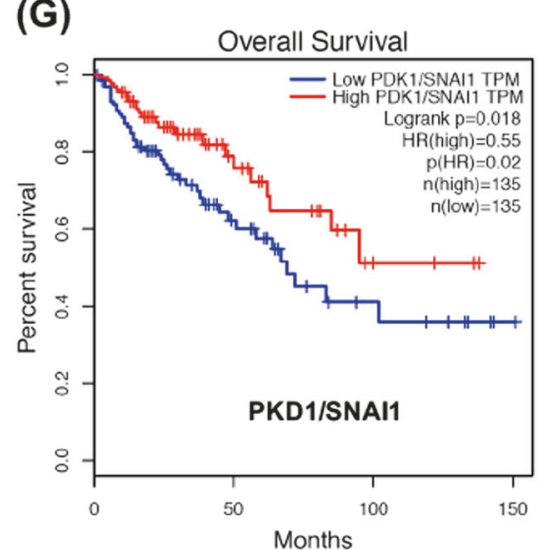

Fig. 5 Clinical characteristics of CDK7 in CRC patients and its association with PKD1/Snail. a Typical immunohistochemical staining for CDK7 expression in stage I IV CRC patient in the commercial tissue microarray; b The Pearson correlation between $C D K 7$ and FN1 in 324 CRC tissues from TCGA data base; $\mathbf{c}$ The Pearson correlation between $C D K 7$ and VIM in 324 CRC tissues from TCGA data base; $\mathbf{d}$ The relative mRNA expression of CDK7 in stage I, II, III, IV CRC patients based on data available from TCGA database; e Overall

\section{Clinical characteristics of CDK7 in CRC patients and its association with PKD1/Snail}

We analyzed the protein expression of CDK7 in tissue microarrays containing 65 samples of CRCs with patientmatched normal mucosal tissues. IHC data showed that the expression of CDK7 was significantly $(p<0.05)$ decreased with the increase of tumor stage in CRC survival (OS) in patients with high $(n=135)$ vs low $(n=135)$ levels of SNAII in CRC patients was plotted by the Kaplan-Meier method; $\mathbf{f}$ Overall survival (OS) in patients with high $(n=135)$ vs low $(n=135)$ levels of CDK7/SNAII in CRC patients was plotted by the Kaplan-Meier method; g Overall survival (OS) in patients with high $(n=135)$ vs low $(n=135)$ levels of PKD1/SNAII in CRC patients was plotted by the Kaplan-Meier method

patients (Fig. 5a, Table S1), suggesting that CDK7 might positively trigger the tumorigenesis while inhibit the metastasis of CRC. As summarized in Table S1, CDK7 expression decreased in CRC patients with higher regional lymph nodes $(\mathrm{N})$, which showed a marginal statistical significance of $p=0.149$. There was no significant difference in the expression of CDK7 expression among ages and sexes (Table S1). 
We assessed the correlation between CDK7 and EMTrelated markers in CRC patients with the data downloaded from LinkedOmics (http://www.linkedomics.org). Although there was no statistical correlation between $C D K 7$ and $C D H 1$ (E-Cad, Figure S5A), the expression of $C D K 7$ was significantly $(p<0.05)$ negatively correlated with the expression of FN1 (Fig. 5b), VIM (Fig. 5c), CDH2, MMP2 and MMP9 (Figure S5 B D) in 324 CRC patients. It suggested that CDK7 might act as a suppressor for motility of cancer cells in CRC patients.

We questioned the possibility of a link between CDK7, PKD1, Snail and CRC clinical development. We further analyzed their expression in 315 samples from TCGA Colorectal data bases. Our results showed that the mRNA expression of $C D K 7$ significantly decreased in CRC tissues with increasing pathologic stages (Fig. 5d). CRC patients with greater number of lymph node metastasis showed less expression of $C D K 7$ mRNA (Figure S5E). Similarly, the expression of $C D K 7$ mRNA decreased with the increase of pathologic $\mathrm{N}$ stage (Figure S5F) and T stage (Figure S5G) of CRC patients. As to the expression of SNAI1, our data showed that CRC patients whose tumors expressing higher SNAII $(n=135)$ showed poorer survival rate as compared with those with less levels $(n=135)$ of SNAI1 $(p=0.08)$ (Fig. 5e). There was no correlation between the mRNA expression of CDK7 and SNAII in CRC tissues (Figure $\mathrm{S} 5 \mathrm{H}$ ). Overall survival rate of CRC patients was independent to the expression level of $C D K 7$ (Figure S5I) or PKD1 (Figure S5J). However, CRC patients whose tumors expressing less CDK7/SNAII $(n=135)$ showed poorer survival rate as compared with those with greater levels ( $n$ $=135, p<0.05$ ) (Fig. 5f). Similarly, CRC patients whose tumors expressing less PKDI/SNAII $(n=135)$ showed poorer survival rate as compared with those with greater levels $(n=135)$ of PKD1/SNAII ( $p<0.01)$ (Fig. 5g). Collectively, our data suggested that CDK7 may contribute to the growth of CRC during tumorigenesis; however, it can suppress the metastasis during cancer development via PKD1/Snail axis.

\section{Discussion}

As a super enhancer inhibitor, THZ1 is a covalent CDK7 inhibitor that can efficiently suppress MYC targets and the MYC driven transcriptional amplification in cancers [10, $12,24]$. It has been reported that THZ1 treatment can significantly inhibit the in vitro and in vivo growth of multiple cancers, including small cell lung cancer [15], oesophageal squamous cell carcinoma [25], tripe negative breast cancer [26], ovarian cancer [27], T-cell lymphomas [28] and neuroblastoma [12]. We herein revealed that the expression of CDK7 in CRC cells and tissues were greater than their corresponding controls. THZ1 can selectively inhibit the in vitro and in vivo growth of CRC cells without the variation of cell cycle but induction of cell apoptosis. As reported, THZ1 treatment did not alter the cell cycle in TNBC [14] and T-cell lymphomas [28] cells. It has been suggested that THZ1 led to a profound induction of apoptosis in high $M Y C N$-expressing cells, but not in cells expressing non-amplified $M Y C N$ [12]. This might be the reason that NCM460, HCT8 and HT29 cells were less sensitive to the treatment of THZ1 in the present study.

One major important finding in our present study was that motility of CRC cells treated with THZ1 was significantly increased. THZ1 treatment can induce EMT-like properties and trigger in vivo metastasis of CRC cells. CDK7 was significantly $(p<0.05)$ negatively correlated with the expression of mesenchymal markers including FN1, VIM and $M M P 2$. Role of CDK7 on cancer metastasis has been reported by one group that $\mathrm{CCNH} / \mathrm{CDK} 7$ can interact with CtBP2 to promote cell migration of breast cancer [29] and esophageal squamous cell carcinoma [30]. In agreement with our findings, recent studies suggested that CDKs controlled the migration and metastatic potential of cancer cells [31]. For example, CDK3 can suppress breast cancer metastasis via inhibiting $\mathrm{Wnt} / \beta$-catenin pathway [32]. Interestingly, CDK3 has also been considered as a tumor promoter, due to its ability of promoting cell growth and transformation [33, 34]. Inhibition of CDK4/6 activity with PD-0332991 suppressed the growth of pancreatic cancer cells, while enhanced their invasion via induction of EMT [35]. Further, depletion of cyclin A2 [36] and cyclin D1 [37] decreased proliferation, but increased migration of cancer cells. THZ1-induced metastasis of CRC cells was consistent with previous viewpoint that two cellular processes, proliferation and migration, do not occur simultaneously, which called "migration-proliferation dichotomy" [38].

We revealed that targeted inhibition of CDK7 can stabilize Snail via downregulation of PKD1. Snail is the first one that reported to repress the $\mathrm{CDH} 1$ gene (encoding Ecadherin) transcription and induce EMT [39, 40]. We found that targeted inhibition of CDK7 recruited protein stability of Snail while it had no effect on its mRNA expression. By screening the factors involved in Snail stability, we found that PKD1, which can phosphorylate Ser11 on Snail [41], was significantly inhibited by THZ1 and si-CDK7. Phosphorylation of Ser11 will trigger the nuclear export of Snail via 14-3-3 $\sigma$ binding and then degradated by E3 ligase FBXO11 via ubiquitylation [23]. As reported, PKD1dependent phosphorylation of Ser11 on SNAG domain is the rate limiting step for FBXO11-mediated Snail protein ubiquitylation and degradation [23]. We confirmed that mutation of Ser11 can attenuate the THZ1-extended protein stability of Snail. Multiple signaling pathways, such as $\beta$ -

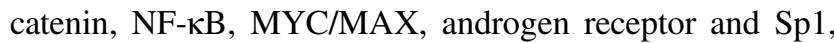


can regulate the transcription of PKD1 in cancer cells via binding with its promoter [42]. As reported, CDK7 inhibition by THZ1 has revealed integral roles for CDK7 in both transcriptional pausing and mRNA cap methylation [43]. A recent study showed that MYC mediates phosphorylation of S5 on the carboxy-terminal domain of RNA Pol II through recruitment of $\mathrm{CDK} 7$, which leads to the activation of targeted transcripts [44]. Whether MYC or other transcription factors are involved in CDK7 regulated transcription of PKD1 via similar approach needs further investigation.

In vivo study and clinical analysis confirmed the suppression effects of CDK7 on the metastasis of CRC. We revealed that expression of CDK7 was elevated in CRC tissue as compared with the adjacent normal tissues. This was consistent with recent reports that CDK7 was increased in cancer tissues [45, 46]. We observed a strong negative correlation of $C D K 7$ and mesenchymal markers including VIM, FN1, N-Cad, MMP2 and MMP9 expression in CRC patients. The expression of CDK7 was negatively correlated with the in vivo metastasis of CRC patients. Although there was no significant difference for the overall survival rate of patients with CDK7 expression level, CRC patients with less CDK7/SNAI1 or PKD1/SNAI1 showed poorer survival rate as compared with those with greater levels. In estrogen receptor positive $(\mathrm{ER}+)$ breast cancer, high-level expression of CDK7 was associated with longer survival in univariate and multivariate analyses [45]. However, patients with high expression of CDK7 exhibited worse overall survival as compared to those with low CDK7 in hepatocellular carcinoma (HCC) [47], gastric [48] and triple negative breast cancer [26]. For an unstratified cohort of 2656 breast cancer patients, no significant association was observed between CDK7 expression and recurrence-free survival [26]. It suggested that the roles of CDK7 on cancer progression might be cancer type specific.

Collectively, our data suggested that CDK7 contributes to the growth of CRC during tumorigenesis. However, CDK7 can suppress the metastasis during cancer development via a PKD1/Snail-dependent manner. The promotive effects on cancer metastasis of THZ1 in the present study imposed great challenge that targeted inhibition of CDK7 is a potential approach for cancer treatment, at least for CRC patients.

\section{Availability of supplementary data}

Detailed methods and materials, Figure S1 to S5, supplementary table S1 to S2 are attached.

Acknowledgements This research was supported by the National Natural Science Foundation of China (Grant No. 81673454, No. 81672608, No. 81472470, No. 31801197, and No. 81572270), the Guangdong Natural Science Funds for Distinguished Young Scholar (No. 2014A030306025), the Hunan Young Talent, China (No.2017RS3051), the Pearl River S\&T Nova Program of Guangzhou (No. 1517000390), and the Fundamental Research Funds for the Central Universities (Sun Yat-sen University) (16ykpy09).

\section{Compliance with ethical standards}

Conflict of interest The authors declare that they have no conflict of interest.

\section{References}

1. Siegel RL, Miller KD, Jemal A. Cancer statistics, 2016. CA. 2016;66:7-30.

2. Kanas GP, Taylor A, Primrose JN, Langeberg WJ, Kelsh MA, Mowat FS, et al. Survival after liver resection in metastatic colorectal cancer: review and meta-analysis of prognostic factors. Clin Epidemiol. 2012;4:283-301.

3. Zullig LL, Smith VA, Jackson GL, Danus S, Schnell M, Lindquist $\mathrm{J}$, et al. Colorectal cancer statistics from the veterans affairs central cancer registry. Clin Colorectal Cancer. 2016;15:e199-204.

4. Restifo NP, Smyth MJ, Snyder A. Acquired resistance to immunotherapy and future challenges. Nat Rev Cancer. 2016;16:121-6.

5. Asghar U, Witkiewicz AK, Turner NC, Knudsen ES. The history and future of targeting cyclin-dependent kinases in cancer therapy. Nat Rev Drug Discov. 2015;14:130-46.

6. Spangler L, Wang X, Conaway JW, Conaway RC, Dvir A. TFIIH action in transcription initiation and promoter escape requires distinct regions of downstream promoter DNA. Proc Natl Acad Sci USA. 2001;98:5544-9.

7. Akoulitchev S, Makela TP, Weinberg RA, Reinberg D. Requirement for TFIIH kinase activity in transcription by RNA polymerase II. Nature. 1995;377:557-60.

8. Bataille AR, Jeronimo C, Jacques PE, Laramee L, Fortin ME, Forest A, et al. A universal RNA polymerase II CTD cycle is orchestrated by complex interplays between kinase, phosphatase, and isomerase enzymes along genes. Mol Cell. 2012;45:158-70.

9. Wong KH, Jin Y, Struhl K. TFIIH phosphorylation of the Pol II CTD stimulates mediator dissociation from the preinitiation complex and promoter escape. Mol Cell. 2014;54:601-12.

10. Kwiatkowski N, Zhang T, Rahl PB, Abraham BJ, Reddy J, Ficarro SB, et al. Targeting transcription regulation in cancer with a covalent CDK7 inhibitor. Nature. 2014;511:616-20.

11. Larochelle S, Amat R, Glover-Cutter K, Sanso M, Zhang C, Allen $\mathrm{JJ}$, et al. Cyclin-dependent kinase control of the initiation-toelongation switch of RNA polymerase II. Nat Struct Mol Biol. 2012;19:1108-15.

12. Chipumuro E, Marco E, Christensen CL, Kwiatkowski N, Zhang $\mathrm{T}$, Hatheway CM, et al. CDK7 inhibition suppresses superenhancer-linked oncogenic transcription in MYCN-driven cancer. Cell. 2014;159:1126-39.

13. Hong SW, Hong SM, Yoo JW, Lee YC, Kim S, Lis JT, et al. Phosphorylation of the RNA polymerase II C-terminal domain by TFIIH kinase is not essential for transcription of Saccharomyces cerevisiae genome. Proc Natl Acad Sci USA. 2009;106: 14276-80.

14. Wang Y, Zhang T, Kwiatkowski N, Abraham BJ, Lee TI, Xie S, et al. CDK7-dependent transcriptional addiction in triple-negative breast cancer. Cell. 2015;163:174-86.

15. Christensen CL, Kwiatkowski N, Abraham BJ, Carretero J, AlShahrour F, Zhang T, et al. Targeting transcriptional addictions in small cell lung cancer with a covalent CDK7 inhibitor. Cancer Cell. 2014;26:909-22.

16. Larochelle S, Merrick KA, Terret ME, Wohlbold L, Barboza NM, Zhang C, et al. Requirements for Cdk7 in the assembly of Cdk1/ 
cyclin B and activation of Cdk2 revealed by chemical genetics in human cells. Mol Cell. 2007;25:839-50.

17. Liang YC, Tsai SH, Chen L, Lin-Shiau SY, Lin JK. Resveratrolinduced G2 arrest through the inhibition of CDK7 and p34CDC2 kinases in colon carcinoma HT29 cells. Biochem Pharmacol. 2003;65:1053-60.

18. Thiery JP, Acloque H, Huang RYJ, Nieto MA. Epithelialmesenchymal transitions in development and disease. Cell. 2009;139:871-90.

19. Davis FM, Stewart TA, Thompson EW, Monteith GR. Targeting EMT in cancer: opportunities for pharmacological intervention. Trends Pharmacol Sci. 2014;35:479-88.

20. Zhou BP, Deng J, Xia W, Xu J, Li YM, Gunduz M, et al. Dual regulation of Snail by GSK-3beta-mediated phosphorylation in control of epithelial-mesenchymal transition. Nat Cell Biol. 2004;6:931-40.

21. Wu Y, Deng J, Rychahou PG, Qiu S, Evers BM, Zhou BP. Stabilization of snail by NF-kappaB is required for inflammation-induced cell migration and invasion. Cancer Cell. 2009;15:416-28.

22. Lamouille S, Xu J, Derynck R. Molecular mechanisms of epithelialmesenchymal transition. Nat Rev Mol Cell Biol. 2014;15:178-96.

23. Zheng H, Shen M, Zha YL, Li W, Wei Y, Blanco MA, et al. PKD1 phosphorylation-dependent degradation of SNAIL by SCFFBXO11 regulates epithelial-mesenchymal transition and metastasis. Cancer Cell. 2014;26:358-73.

24. Loven J, Hoke HA, Lin CY, Lau A, Orlando DA, Vakoc CR, et al. Selective inhibition of tumor oncogenes by disruption of superenhancers. Cell. 2013;153:320-34.

25. Jiang YY, Lin DC, Mayakonda A, Hazawa M, Ding LW, Chien WW, et al. Targeting super-enhancer-associated oncogenes in oesophageal squamous cell carcinoma. Gut. 2017;66:1358-68.

26. Li B, Ni Chonghaile T, Fan Y, Madden SF, Klinger R, O’Connor $\mathrm{AE}$, et al. Therapeutic rationale to target highly expressed CDK7 conferring poor outcomes in triple-negative breast cancer. Cancer Res. 2017;77:3834-45.

27. Francavilla C, Lupia M, Tsafou K, Villa A, Kowalczyk K, Rakownikow Jersie-Christensen R, et al. Phosphoproteomics of primary cells reveals druggable kinase signatures in ovarian cancer. Cell Rep. 2017;18:3242-56.

28. Cayrol F, Praditsuktavorn P, Fernando TM, Kwiatkowski N, Marullo R, Calvo-Vidal MN, et al. THZ1 targeting CDK7 suppresses STAT transcriptional activity and sensitizes T-cell lymphomas to BCL2 inhibitors. Nat Commun. 2017;8:14290.

29. Wang Y, Liu F, Mao F, Hang Q, Huang X, He S, et al. Interaction with cyclin $\mathrm{H} /$ cyclin-dependent kinase 7 (CCNH/CDK7) stabilizes C-terminal binding protein 2 (CtBP2) and promotes cancer cell migration. J Biol Chem. 2013;288:9028-34.

30. Zhang J, Zhu J, Yang L, Guan C, Ni R, Wang Y, et al. Interaction with $\mathrm{CCNH} / \mathrm{CDK} 7$ facilitates $\mathrm{CtBP} 2$ promoting esophageal squamous cell carcinoma (ESCC) metastasis via upregulating epithelial-mesenchymal transition (EMT) progression. Tumour Biol. 2015;36:6701-14.

31. Roesley SN, Suryadinata R, Morrish E, Tan AR, Issa SM, Oakhill JS, et al. Cyclin-dependent kinase-mediated phosphorylation of breast cancer metastasis suppressor 1 (BRMS1) affects cell migration. Cell Cycle. 2016;15:137-51.

32. Cao T, Xiao T, Huang G, Xu Y, Zhu JJ, Wang K, et al. CDK3, target of miR-4469, suppresses breast cancer metastasis via inhibiting Wnt/beta-catenin pathway. Oncotarget. 2017;8:84917-27.
33. Zheng D, Cho YY, Lau AT, Zhang J, Ma WY, Bode AM, et al. Cyclin-dependent kinase 3-mediated activating transcription factor 1 phosphorylation enhances cell transformation. Cancer Res. 2008;68:7650-60.

34. Xiao T, Zhu JJ, Huang S, Peng C, He S, Du J, et al. Phosphorylation of NFAT3 by CDK3 induces cell transformation and promotes tumor growth in skin cancer. Oncogene. 2017;36:2835-45.

35. Liu F, Korc M. Cdk4/6 inhibition induces epithelial-mesenchymal transition and enhances invasiveness in pancreatic cancer cells. Mol Cancer Ther. 2012;11:2138-48.

36. Arsic N, Bendris N, Peter M, Begon-Pescia C, Rebouissou C, Gadea $\mathrm{G}$, et al. A novel function for Cyclin A2: control of cell invasion via RhoA signaling. J Cell Biol. 2012;196:147-62.

37. Lehn S, Tobin NP, Berglund P, Nilsson K, Sims AH, Jirstrom K, et al. Down-regulation of the oncogene cyclin D1 increases migratory capacity in breast cancer and is linked to unfavorable prognostic features. Am J Pathol. 2010;177:2886-97.

38. Bhandari D, Lopez-Sanchez I, To A, Lo IC, Aznar N, Leyme A, et al. Cyclin-dependent kinase 5 activates guanine nucleotide exchange factor GIV/Girdin to orchestrate migration-proliferation dichotomy. Proc Natl Acad Sci USA. 2015;112:E4874-83.

39. Batlle E, Sancho E, Franci C, Dominguez D, Monfar M, Baulida $\mathrm{J}$, et al. The transcription factor Snail is a repressor of E-cadherin gene expression in epithelial tumour cells. Nat Cell Biol. 2000;2:84-89.

40. Cano A, Perez-Moreno MA, Rodrigo I, Locascio A, Blanco MJ, del Barrio MG, et al. The transcription factor Snail controls epithelial-mesenchymal transitions by repressing E-cadherin expression. Nat Cell Biol. 2000;2:76-83.

41. Du C, Zhang CY, Hassan S, Biswas MHU, Balaji KC. Protein kinase D1 suppresses epithelial-to-mesenchymal transition through phosphorylation of snail. Cancer Res. 2010;70:7810-9.

42. Nickkholgh B, Sittadjody S, Rothberg MB, Fang XL, Li KZ, Chou JW, et al. Beta-catenin represses protein kinase D1 gene expression by non-canonical pathway through MYC/MAX transcription complex in prostate cancer. Oncotarget. 2017;8:78811-24.

43. Nilson KA, Guo JN, Turek ME, Brogie JE, Delaney E, Luse DS, et al. THZ1 reveals roles for Cdk7 in co-transcriptional capping and pausing. Mol Cell. 2015;59:576-87.

44. Posternak V, Ung MH, Cheng C, Cole MD. MYC Mediates mRNA cap methylation of canonical Wnt/beta-catenin signaling transcripts by recruiting CDK7 and RNA methyltransferase. Mol Cancer Res. 2017;15:213-24.

45. Patel H, Abduljabbar R, Lai CF, Periyasamy M, Harrod A, Gemma C, et al. Expression of CDK7, cyclin H, and MAT1 is elevated in breast cancer and is prognostic in estrogen receptorpositive breast cancer. Clin Cancer Res. 2016;22:5929-38.

46. Bartkova J, Zemanova M, Bartek J. Expression of CDK7/CAK in normal and tumor cells of diverse histogenesis, cell-cycle position and differentiation. Int J Cancer. 1996;66:732-7.

47. Wang C, Jin H, Gao D, Wang L, Evers B, Xue Z, et al. A CRISPR screen identifies CDK7 as a therapeutic target in hepatocellular carcinoma. Cell Res. 2018;28:690.

48. Wang Q, Li M, Zhang X, Huang H, Huang J, Ke J, et al. Upregulation of CDK7 in gastric cancer cell promotes tumor cell proliferation and predicts poor prognosis. Exp Mol Pathol. 2016;100:514-21. 